\title{
Research on Brain Has a Negative Bias and How to Fix It

\author{
Yue Cui*
}

\author{
Qingdao No.2 Middle School of Shandong Province \\ *Corresponding author. Email: 2175601165@qq.com
}

\begin{abstract}
Scientists hypothesize that there is a general bias towards negative entities (e.g., events, objects, personal characteristics) based on innate tendencies and experiences. This article mainly studies why our brain has negative bias and how we can overcome it. At present, psychologists have studied the relationship between the brain and negative bias, the factors that affect the strength of negative bias, and the threat of negative bias to health. For example, when you drive to work, someone interrupts you when you get off the highway. Suddenly, a car rushes out and you slam on the brake. It's enough to make you feel terrible all day. So, why does cutting off a small thing have such a big impact on us? Why does a negative experience ruin a good day? According to the research, scientists come to the conclusion that the tendency that we are more likely to record negative events, and we put forward suggestions to overcome negative bias from many aspects is named negative bias. In this case, it is important for us to know about negative bias. In this article, we mainly explain and study what negative bias is; why people have negative prejudice from perspective of gene, people development, and brain response; the impact on us of negative bias in relationships with others, anxiety, decision making, and perception of others; and how to overcome it.
\end{abstract}

Keywords: Negative Bias, Psychological research, Brain research.

\section{INTRODUCTION}

Negative bias, that is, even when the intensity is equal, more negative things (such as unpleasant thoughts, emotions, or social interactions; harmful/traumatic events have a greater impact on a person's mental state and process than neutral or positive things. In other words, very positive things usually have less impact on a person's behavior and cognition than the same emotional but negative things. It's our tendency not only to record negative stimuli more easily, but also to stay on these events. Also known as positive and negative asymmetry, this negative bias means that we feel the sting of condemnation more strongly than the joy of praise. This psychological phenomenon explains why bad first impressions are so difficult to overcome, and why past trauma has such a lasting impact. In almost all interactions, we are more likely to notice negative things and remember them more vividly. Negative bias has been studied in many different fields, including impression formation and general assessment; Attention, learning and memory; And decision making and risk considerations. According to research, as human beings, we tend to remember traumatic experiences more easily than positive experiences, recall insults more than praise, respond more strongly to negative stimuli, think about negative things more frequently than positive things, and respond more strongly to negative time than to the same positive events. For example, you may have a good day at work when a colleague makes a casual comment that annoys you. Then you'll find yourself worrying about his words for the rest of your workday. When you come home from work and are asked how you are doing today, you reply that it's terrible - despite a negative event, it's generally good. Another example, Dr. Bono explains, "Denny Kahneman, an economist who won the 2002 Nobel Prize for his work, designed studies that allowed participants to imagine either losing $\$ 50$ or winning $\$ 50$. Even if the amount was the same, the emotional response was much greater for those who imagined what it would be like to lose money. In other words, the negativity of losing something is far greater than the benefit of acquiring something, even if the "something" lost or acquired is objectively equivalent. This negative bias leads people to pay more attention to the bad things that happen, making them seem more important than they really are. 


\section{THE EFFECTS OF NEGATIVE BIAS}

Although we may no longer need to be as vigilant as our early ancestors were in order to survive, negative bias still plays an important role in the way our brains work. Studies have shown that negative bias has a variety of effects on people's thinking, reaction and feelings. People may feel the consequences of this bias in everyday areas, including relationships, emotions, decision-making, and your perception of others.

Bias in relationships: we know that negative bias can affect our impressions of other people, our decisions, and our attention. Therefore, it can influence our relationship with others in many ways. Among them, it may lead us to make the worst assumptions about people we don't know yet. Our beliefs and expectations influence our subsequent interactions with them [1]. By making pessimistic assumptions about another person's reaction, we may also fall into the trap of letting our (groundless) attitude influence our behavior; And according to the concept of negative effect, prejudice leads us to interpret negative events as more important than positive ones. Compared with their positive behavior, we can regard someone's mistakes as highly prominent, which leads us to reflect on them and damage our relationship.

Bias in anxiety: being more sensitive to negative information can also affect our neural circuits. Williams and colleagues studied these effects in their 2009 paper: negative bias in the risk of depression and anxiety. Some effects of negative bias may include an increase in heart rate during fear perception and a higher fear response, both of which are associated with anxiety [2]. In this study, the authors also provide evidence that self-reported negative bias measures are associated with depression and anxiety, leading to sleep disorder, which have been lined to obesity or cardiovascular disease. The result suggests that although this phenomenon may be evolutionary, it is not without cost

Decision making: by focusing on or overemphasizing the potential negative effects of decision-making, research shows that we are more likely to avoid risk [3]. Therefore, when faced with choices with potential benefits and risks, we tend to think more about the latter - a good example, that is, negative effectiveness in action [4]. In the rapidly changing, changeable and often ambiguous competitive environment, this will significantly affect the competitive advantage. From an organizational point of view, we can see Kodak, once a leading photographic film company in the world. By choosing to continue to focus on its core "strength" (photographic film) instead of exploring the growing trend of digital photography, it lost its competitive position to its competitors Sony, Canon and Fujifilm before filing for bankruptcy in 2002 . When imagining a scenario involving getting a certain amount of money or losing the same amount of money, the risk of loss lies in the desire for positive returns. Even if the two possibilities are equivalent, people's negative reaction to losing $\$ 50$ is stronger than the positive emotion when they get $\$ 50$.

Perception of others: when forming an impression of others, people also tend to pay more attention to negative information. For example, studies have shown that when both "good" and "bad" adjectives are given to describe another person's personality, participants pay more attention to the bad adjectives when they form the first impression. We have considered how the formation of impressions affects our relationships with others, which applies to the workplace. Effective collaboration, teamwork and continuous professional development all depend on our ability to get along with others and interact in a positive way to achieve common goals. By making us focus and focus on negative entities, bias makes it harder for us to accept constructive feedback, encourage others, and build trust with colleagues. Research on this shows that we can start to address negative bias in the workplace by increasing the ratio of positive comments to negative comments we give [5]. In order to improve team performance and lead others more effectively, in other words, a good target ratio is 5:6:1. Give it a try!

\section{WHY PEOPLE HAVE NEGATIVE IMPACT}

"Bad things are easier to believe. Have you noticed that? " Said Vivian, played by Julia Roberts in pretty women (1990). 1 It turns out that Roberts' character Vivian touches upon an unfortunate psychological truth" It's really easier to believe "bad stuff" for reasons that may surprise you. First, our tendency to focus on negative rather than positive information evolved from our cave ancestors. At that time, alertness to danger, or "bad things", was a matter of life and death. Those who are more sensitive to danger and more concerned about the bad things around them are more likely to survive" Our inherited genes predispose us to pay special attention to the negative aspects of the environment that may be harmful to us, "explains psychologist and happiness researcher Dr. Timothy J. Bono, who teaches happiness science at St. Louis University in Washington. In this way, indulging in "bad things" is similar to the feeling of pain - it's our bodies trying to protect our safety. In addition, negative emotions wake up the amygdala, the amygdala like brain structure that psychologist Rick Hansen, founder of wellspring Institute of neuroscience and meditation intelligence, calls "your brain's wake-up call.". According to Dr Hansen, the amygdala "uses about two-thirds of the neurons to look for bad news. Once the alarm goes off, negative events and experiences will be 
quickly stored in memory, while positive events and experiences usually need more than ten seconds or more of consciousness to be transferred from the short-term memory buffer to the long-term memory buffer. " In addition, in 2010, a video spread wildly on the Internet, vividly showing the attention bias. The video asked the audience to count the number of passes by the person in the white shirt. The task is a farce. When people are immersed in a slightly difficult counting task, a gorilla will walk through the middle of the video. However, most people never notice it. When asked what they saw, most viewers were proud to announce the numbers they came to - and didn't realize until they looked back that they had missed something that should have been obvious. Mack and Rock's 2 research shows that while our brain may process what our eyes see, it may never be aware of it. Your attention and attention is the key to information processing, it filters out conscious thought. So, negative bias is actually a negative attention bias. When we focus on the negative, we actually reshape our perception to see the negative. You may be so focused on all the neve events in your computing life that you completely miss the positive gorilla. [6]

Development: studies show that this negative bias begins to appear in infancy. Very young babies tend to pay more attention to positive facial expressions and tones3, but that starts to change as they approach the age of one. Brain research shows that around this time, babies begin to respond more to negative stimuli. This suggests that the brain's negative bias occurs in the second half of the first year after a child is born. There is some evidence that this bias may actually begin at the development stage. A study has found that infants as young as three months show signs of negative bias in their social evaluation of others. [7]

Brain response: neuroscience evidence suggests that the brain is more responsive to negative stimuli. Studies involving the measurement of event-related brain potentials (ERP) have shown that the brain responds to specific sensory, cognitive or motor stimuli, indicating that negative stimuli cause greater brain responses than positive stimuli. In a study by psychologist John Cacioppo, participants were shown pictures of positive, negative or neutral images. The researchers then looked at electrical activity in the brain. Compared with positive or neutral images, negative images produced stronger responses in the cerebral cortex. Take the research of Dr. John Cacioppo 4 as an example. He showed people pictures that are known to cause positive emotions (such as Ferrari or pizza), pictures that are sure to cause negative emotions (a broken face or a dead cat), and pictures that are known to cause neutral emotions (plates, fans). At the same time, he recorded the electrical activity of the brain. The cerebral cortex reflects the degree of information processing. Kachobo showed that the brain responded more strongly to what it thought was a negative stimulus. There's a bigger surge in electrical activity. Therefore, pessimistic news can influence our attitude more than good news.[8]

\section{HOW TO FIX NEGATIVE BIAS}

We may think that the best way to overcome one bias is to use it with a positive bias. Isn't it better to serve your happiness by providing it with positive feelings and information rather than negative information? But it's not that simple. Negative events hurt you more than positive events help you. However, encouraging positive bias makes you more likely to avoid negative events or experience positive emotions. In fact, it can backfire. Positive bias is similar to the more well-known term confirmation bias 5. When people prefer positive confirmation, they are less likely to notice or accept negative information. We start each day with expectations and expect the world to realize it. If it doesn't, we'll always find a way to sense its existence and think it will come true. You may be in a higher mood but you'll be at a higher risk before you know it. Like very optimistic gamblers. They can spend all their money to pursue positive events that they are absolutely sure will happen. When they make mistakes a dozen times in a row, positive bias redefines them as: "Oh, it means my lucky chance is coming!" Or, for example, an ordinary worker put into an ordinary job. Positive bias may lead them to believe that they are making every effort to succeed in their work. Without a little doubt, a little self doubt, or even a little negativity, they may never find it necessary to work harder or in a different way. If they go to work every day expecting it to move in one direction and distort their efforts to confirm that expectation, they may miss opportunities. Positive bias is not feasible, but psychologists have come up with other ways to overcome negative bias. [9]

Enjoy positive moments because it takes more time to remember positive experiences, so it's important to pay extra attention to the good things that happen. The negative things may be quickly transmitted and stored in your long-term memory, you need to make more efforts to get the same effect of happy time. So when something great happens, take the time to really focus on it. Replay the moment many times in your memory and focus on the wonderful feeling it evokes.

Have you ever noticed that solving a challenging problem will make you forget the slight pain? It turns out that we can get rid of negative emotions by transferring our mental energy to other places, such as in jigsaw puzzles or memory games. Distraction allows you to refocus your attention on negative events that may have 
a disproportionate impact on your ability to process information. For example, being criticized at work, although bad, may make you look at your work in a negative light for a few weeks. Instead of dwelling on this fact, we should divert our attention. If you can separate yourself from a negative event, even if it's temporary, you can make room between you and its impact on you. That space gives you perspective. Distraction is a powerful tool that can even be used to help treat PTSD 6 symptoms. However, the key is not to use distraction to avoid negative emotions. Negative events are a natural part of life. Escaping them with unconscious interference will only make things worse. But a healthy distraction can give you the space you need to think clearly and be more productive.

Keep a gratitude diary, although it sounds weird, tacky and stupid. But research shows that it's very effective. NPR reports on a large number of studies show that practicing gratitude can have a variety of positive effects. Regular gratitude and attention to the good things in life can improve sleep, reduce stress and promote interpersonal relationships. Practical gratitude is one of the most useful research achievements in the field of positive psychology. Although your instincts may be cynical, quantifying the positivity in your life, writing these things down physically, and developing the habit of doing so over and over again can slowly retrain your mind and keep away from negative prejudices. Robert Emmons7 offers some tips on keeping a gratitude diary. They include: focusing on people rather than things, tasting surprise activities, writing only once or twice a week but with depth

Mindfulness: breathing, meditation and other exercises mindfulness is a good way to better adapt to their emotions (charoensukmongkol, 2015). Through guided meditation, reflection, and other mindfulness interventions, you can begin to observe your feelings and thoughts more objectively. More promising evidence comes from a 2011 study by the authors kiken and hook, who found that when participants practice mindfulness breathing, positive judgment increases and optimism increases. Compared with the control group, these participants performed better in the tests that needed to classify positive stimuli, which led researchers to believe that mindfulness practice can have a significant positive impact on bias (kiken \& hook, 2011).

\section{CONCLUSION}

Through the artical, we can know that because it is easier for people to record negative stimuli and stay on these events, we are immersed in the episode of someone interrupting our driving in the morning all day. In order to overcome this situation as much as possible, we can overcome the negative bias by distracting our attention and writing gratitude diary.

\section{REFERENCES}

[1] Stacey Wood \& Michael A. Kisley. The Negativity Bias Is Eliminated in Older Adults: Age-Related Reduction in Event-Related Brain Potentials Associated With Evaluative Categorization. Psychology and Aging 2006, Vol. 21, No. 4, 815820

[2] HE Marano. Our brain's negative bias. - Psychology Today, 2003 - tmctraining.net.

[3] Paul Rozin, Edward B. Royzman. Negativity Bias, Negativity Dominance, and Contagion. Personality and Social Psychology Review by 2001, Vol. 5, No. 4, 296-320.

[4] If You Feel Thankful, Write It Down. It's Good For Your Health. December 24, 20185:01 AM ET Heard on Morning Edition.

[5] Hamlin JK, Wynn K, Bloom P. Three-month-olds show a negativity bias in their social evaluations. Dev Sci. 2010;13(6):923-929. doi:10.1111/j.1467-7687.2010.00951.x.

[6] Ito TA, Larsen JT, Smith NK, Cacioppo JT. Negative information weighs more heavily on the brain: The negativity bias in evaluative categorizations. J Pers Soc Psychol. 1998;75(4):887-900.

[7] Kahneman D, Tversky A. Choices, values, and frames. American Psychologist. 1984;39(4):341350. doi:10.1037/0003-066x.39.4.341.

[8] Alberini CM. Long-term memories: The good, the bad, and the ugly. Cerebrum. 2010;21.

[9] Cacioppo JT, Cacioppo S, Gollan JK. The negativity bias: Conceptualization, quantification, and individual differences. Behavioral and Brain Sciences. 2014;37(3):309-310. 